\title{
Viel Lärm um Nichts!
}

l n der Überschrift einer medizinischen Publikation stand vor kurzem lakonisch: „Enttäuschend - Antibiotika zur Sekundärprävention bei KHK.“ Dieser Satz fasst das (vorläufige) Ende einer der umstrittensten Theorien der Atheroskleroseentstehung und -therapie zusammen - nämlich die Hypothese, dass infektiöse Vorgänge zur Entstehung der Atherosklerose beitragen und, konsequenterweise, eine antiinfektiöse Therapie den Ablauf der Atherosklerose beeinflussen könnte oder sogar müsste.

Was steckt dahinter? Heute wissen wir, dass das akute Koronarsyndrom (ACS) nicht notwendigerweise dadurch entsteht, dass das Lumen des Koronargefäßes sukzessive enger wird, bis es zum Verschluss kommt. Mehr als die Hälfte aller Koronarereignisse entstehen bei Stenosen, die weniger als 50\% des Lumens einengen. Auslöser des akuten Koronarsyndroms ist eine Ruptur der Kappe über der Plaque und der daraus resultierende thrombotische Verschluss. Diese Ruptur ist das letzte Glied der komplexen Ereigniskette, die zur Entstehung atherosklerotischer Plaques führt. Ungeklärt und weltweit kontrovers diskutiert ist in diesem Zusammenhang die Rolle des hsCRP („high-sensity c-reactive protein“). Ob eine Beeinflussung dieses Parameters - zum Beispiel durch eine Statintherapie - die Prognose der Patienten verbessern kann, ist jedoch noch nicht belegt.

Bei der Diskussion über die Rolle der Entzündung kam die Infektionstheorie ins Spiel: Demnach könnten bakterielle Infektionen Entzündungsvorgänge in der Plaque anstoßen oder unterhalten. Bei der Suche nach infektiösen Agenzien wurde - nach dem Ausschluss anderer Keime (z.B. Helicobacter pylori) - eine Infektion mit Chlamydien als potenzielle Ursache vermutet. Insbesondere in Deutschland fand diese Hypothese durch die Unterstützung führender Infektiologen eine unverständlich große Publizität - unverständlich deshalb, weil die der damaligen Kampagne zugrunde liegenden Studien aus Argentinien und England aufgrund ihres ungenügenden Studiendesigns, geringer Patientenzahlen und kurzer Laufzeiten nicht den Anforderungen einer evidenzbasierten Medizin entsprachen.

Inzwischen ist die Datenlage eindeutig. Bis vor kurzem lagen Studien bei mehr als 2000 Patienten vor, die mit Makroliden bzw. Chinolonen behandelt wurden ohne jedes Ergebnis. In diesem Jahr sind mit der ACESStudie („Azithromycin and Coronary Events“; N Engl J Med 2005; 352: 1637-1645) und der PROVE-IT-TIMIStudie („Atorvastatin Evaluation and Infection Therapy Thrombolysis in Myocardial Infarction“; N Engl J Med 2005; 352: 1646-1654) zwei weitere Untersuchungen mit Azithromycin bzw. Gatifloxazin ebenfalls mit negativem Ergebnis abgeschlossen worden. Natürlich bleiben Fragen offen, zum Beispiel nach der Spezifität der ver- wendeten Antibiotika und der Dauer der antibiotischen Behandlung, klar ist aber, dass nach dem heutigen Wissensstand eine antibiotische Therapie in der Sekundärprävention der Atherosklerose nicht gerechtfertigt ist.

Man könnte die Antibiotikatherapie jetzt als Fußnote in der Medizingeschichte abhaken. Allerdings kann man nicht so einfach zur Tagesordnung übergehen. Viele Patienten, die über die schwache Basis dieser Strategie nicht unterrichtet sein konnten, haben ihre Langzeitmedikation mit Lipidsenkern bzw. Antithrombotika mit der Hoffnung abgesetzt, mit der kurzzeitigen Einnahme von Antibiotika eine Alternative zu erhalten. Ob dadurch kurz- oder langfristig Schäden entstanden sind, lässt sich nicht abschätzen - man muss aber aus diesem Irrweg lernen, dass therapeutische Empfehlungen für Volkskrankheiten wie die Atherosklerose nur nach gründlicher Prüfung der Daten ausgesprochen werden sollten.

Die Konsequenz aus dem Misserfolg der Antibiose besteht im konsequenten Einsatz der erprobten Mittel: Die Reduktion des Blutdrucks um $1 \mathrm{mmHg}$ senkt das Langzeitrisiko für den Myokardinfarkt um 2-3\%, eine 10\%ige Reduktion des LDL-Cholesterins verringert das Risiko für kardiovaskuläre Todesfälle um $10 \%$ und für kardiovaskuläre Ereignisse um 25\%. Eine Beendigung des Rauchens halbiert das kardiovaskuläre Risiko innerhalb weniger Jahre, ähnliches gilt für eine konsequente Behandlung des Diabetes mellitus und des Übergewichts. Hier bestehen noch große Defizite in der Umsetzung gesicherter wissenschaftlicher Ergebnisse in die tägliche klinische Praxis, die abgebaut werden sollten, ehe wir uns auf unsicheres Terrain begeben!

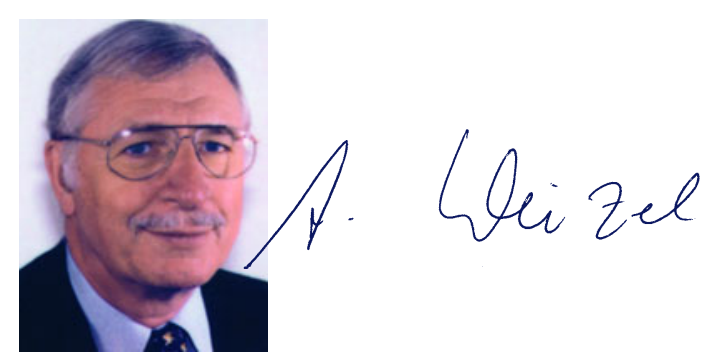

Prof. Dr. A. Weizel, Mannheim 